\title{
How many fund managers does a fund-of-funds need?
}

Received (in revised form): 20th March, 2008

\section{Kartik Patel}

is a senior risk associate with Prisma Capital Partners, a fund of hedge funds. At Prisma he has been involved with building a proprietary system of risk tools used for portfolio construction, strategy allocation and for analysing individual hedge funds. He holds an M.S. in financial engineering from Columbia University, M.S. in electrical engineering from University of Maryland and a B-Tech in electrical engineering from I.I.T. Bombay.

\begin{abstract}
We investigate the appropriate number of hedge funds to be held in a fund-of-funds portfolio with the purpose of meeting a typical benchmark mandated by institutional investors. The technique of random sampling from a universe of managers in the Credit Suisse/Tremont hedge fund index is used to generate portfolios. We use naïve diversification as well as strategy diversification methods, where the latter simulates a diversified fund-of-funds portfolio. We conclude that 40 managers are sufficient to beat the chosen benchmark.
\end{abstract}

Pensions (2008) 13, 61-69. doi:10.1057/pm.2008.9

Keywords: fund-of-funds, portfolio of hedge funds, optimal number of managers, naïve diversification, strategy diversification

\section{Introduction}

Over the past several years investments in the hedge fund asset class have increased dramatically. According to Hedge Fund Research's 2007 yearend report, the total assets managed by hedge funds have increased from $\$ 490 \mathrm{bn}$ in the year 2000 to $\$ 1,868$ bn in 2007 . Interestingly, the total assets managed by fund-of-funds ( $\$ 798 \mathrm{bn}$ ) now account for about 42 per cent of the total assets managed by hedge funds. The assets managed by fund-of-funds have increased nine-fold since 2000 , when it represented only 16 per cent of the total hedge fund assets. In a recent report published by Preqin Hedge, ${ }^{1} 60$ per cent of the capital invested in fund-of-funds come from institutional investors. The reason for the increased popularity of hedge funds among institutional investors is their ability to generate high risk adjusted returns while at the same time having a low correlation to traditional asset classes such as

Correspondence: Kartik Patel, Prisma Capital Partners, Harborside Financial Center, 208 Plaza Ten, Jersey City, NJ 07311, USA.

Tel: +1 201356 7023;

Fax: +1 201356 7010;

E-mail: kpatel@Prismapartners.com stocks and bonds. Investing via fund-of-funds give investors exposure to a diversified set of hedge fund managers following different strategies (like long/short equity, convertible arbitrage, etc). A majority of fund-of-funds have a diversified approach whereby investment is made in multiple hedge fund strategies, although there are fund-offunds which are strategy specific.

Selecting managers in the portfolio is obviously very critical to the performance of the portfolio, but at the same time it is important to decide upon the number of managers needed in the portfolio. Since Markowitz, ${ }^{2}$ portfolio diversification has been a traditional way of reducing risk. In particular, the optimal number of securities in a portfolio has been widely debated, more recently for example by Statman ${ }^{3}$ for stocks and by L'habitant and Learned ${ }^{4}$ and Amo et al. ${ }^{5}$ for hedge funds. Increasing the number of securities (in our case hedge fund managers) in a portfolio results in lower volatility if the securities have low correlation with each other, but the price of lower volatility is usually lower return. Here we investigate the appropriate number of hedge funds in a portfolio from the 
perspective of an institutional diversified fundof-funds investment management mandate.

Typically the benchmark given to an absolute return mandate fund-of-funds by its investors ranges from T-Bill to T-Bill +5 per cent, with an incentive fee for out performance. In addition, many fund-of-funds investors are involved in a portable alpha swap, which obligates them to pay the 'risk-free' rate, ${ }^{6}$ so it is important that they at least earn T-Bill returns. A fund-of-funds management firm is more averse to the risk of underperforming the benchmark than it is enthusiastic about the chance of obtaining higher returns. We consider a hypothetical benchmark of T-Bill +2.5 per cent, which falls inside the range of typical benchmarks used.

The risk of underperforming the benchmark is the main risk we consider in this paper. With this objective in mind, we study the risk of underperforming the benchmark as a function of the number of managers in the portfolio. In order to study the risk return characteristics of portfolios of different sizes, we randomly generate portfolios from the selected universe via simulation. The universe of hedge funds that we use is constructed from the managers in the Credit Suisse/Tremont hedge fund index. The reason for choosing this manager set is because the index rules for inclusion are similar to what an institutional investor would require (as we discuss later).

A portfolio of size $M$ is simulated by randomly drawing $M$ funds from the universe. Hundreds of such portfolios are created for a selected size $M$, for the years 2002-2006, and the probability of annual performance exceeding the benchmark for the corresponding year is computed. We will show that as $M$ increases, the probability of outperforming the benchmark increases, while at the same time the probability of generating higher returns decreases.
We consider two methods of simulation: naïve diversification and strategy diversification. Naïve diversification is implemented via simple random sampling whereby the strategy type of a randomly selected fund is ignored when making the selection. To simulate performance of a typical fund-of-funds portfolio that is diversified across different strategies, we use the method of strategy diversification implemented via stratified random sampling whereby we constrain the number of funds to be drawn from each strategy. We compare the performance of portfolios generated from the two methods of simulation.

We conclude that a portfolio of approximately 40 funds is appropriate for outperforming the benchmark with a high confidence level.

\section{Benchmark}

As mentioned above, the hypothetical benchmark that we consider is T-Bill +2.5 per cent, which is shown in Table 1 for different years. Note that the returns reported in the remainder of the paper are all in excess of the benchmark returns.

\section{Universe}

We use the managers in the Credit Suisse/ Tremont hedge fund index as our initial universe. The reason for selecting the CS/Tremont index as opposed to other indices like HFR (Hedge Fund Research) index is that the former has stricter inclusion requirements (eg a fund needs to have AUM of at least $\$ 50 \mathrm{~m}$ to be considered to be part of the index and a fund needs to have at least one year of track record), ${ }^{7}$ similar to the requirements of a typical institutional investor.

The universe is created by identifying the funds in the CS/Tremont index from the Lipper TASS and HFR hedge fund databases. We only include funds with full five-year track records from 2002 to 2006 in the universe (totaling 254). ${ }^{8}$

Table 1: Benchmark returns for different years

\begin{tabular}{llllll}
\hline & $2002(\%)$ & $2003(\%)$ & $2004(\%)$ & $2005(\%)$ & $2006(\%)$ \\
\hline T-Bill $^{\mathrm{a}}$ & 1.8 & 1.2 & 1.3 & 3.1 & 4.9 \\
Benchmark (T-Bill+2.5\%) & 4.3 & 3.7 & 3.8 & 5.6 & 7.4 \\
\hline
\end{tabular}

aAnnual returns of three month T-Bills

Source: Merrill Lynch. 
Table 2 shows the strategy constitution of the universe. As can be seen from the table, the universe is skewed by the number of long-short managers. Table 3(a) tabulates the average excess returns of the funds in the universe for different years, while Table $3(\mathrm{~b})$ tabulates the average excess returns of the funds strategy by strategy. If we choose to invest in all 254 funds equally we would get the returns shown in Table 3(a) and since the excess returns (over the benchmark) for all years are positive, we would attain our objective of beating the benchmark. Alternatively, if we want to diversify across different strategies, we can invest equally in all funds within each strategy, and invest equal dollar amounts per

Table 2: Universe composition

\begin{tabular}{lcc}
\hline Strategy & $\begin{array}{c}\text { Number of } \\
\text { funds }\end{array}$ & Percentage \\
\hline 1 Convertible arbitrage & 5 & 2 \\
2 Event & 24 & 9 \\
3 Equity market neutral & 17 & 7 \\
4 Fixed income & 18 & 7 \\
5 Global macro & 8 & 3 \\
6 Long short & 107 & 42 \\
7 Managed futures & 24 & 9 \\
8 Multi strategy & 17 & 7 \\
9 Short bias & 5 & 2 \\
10 Emerging market & 29 & 11 \\
& & \\
Total & 254 & \\
\hline
\end{tabular}

strategy, and would get returns shown in Table $3(\mathrm{~b})$, and this way too we would be able to beat the benchmark. But the question is whether we can invest in fewer funds, thus offering higher return potential and still be able to meet the benchmark with a high degree of confidence. We address this question by randomly creating portfolios of different sizes, and present the methodology and results in the following sections.

\section{Methodology of random sampling}

We study the impact of varying the number of funds $(M)$ in a portfolio by the technique of random sampling. We use simple and stratified random sampling to create portfolios as described below.

\section{Naïve diversification}

We randomly select $M$ funds from the universe and equally weight them to create a portfolio. In this method the strategy type or style of the managers selected is ignored while making the selection, so we could end up with a portfolio of managers of the same style. Although one can select $M$-fund portfolios from a population of size $N$ in $N$-choose- $M$ possible ways $\left({ }^{N} C_{M}\right)$, we restrict the sample size to be 1,000 . Our

Table 3: Universe average returns for different years

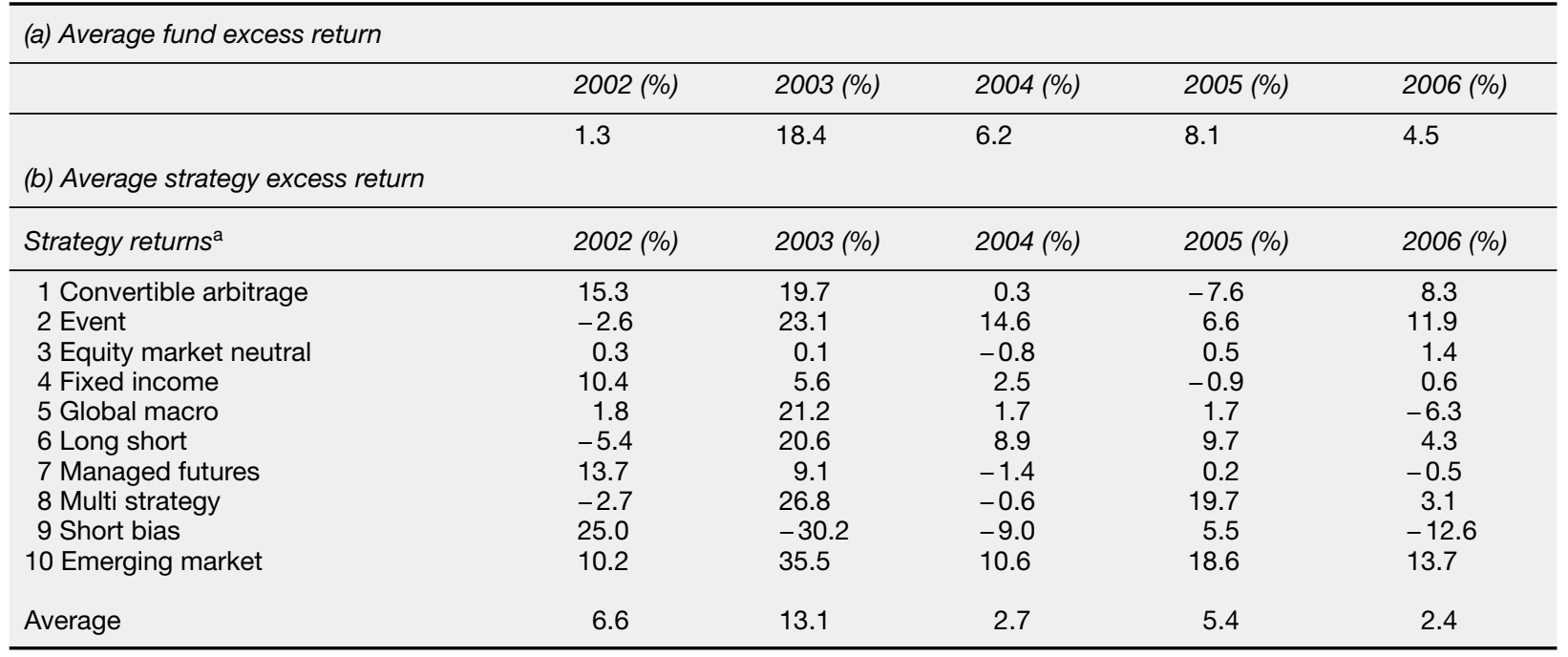

${ }^{a}$ Funds within each strategy are equally weighted to obtain the strategy returns. 
construction algorithm proceeds for each year between 2002 and 2006 as follows:

1. Draw ten $(M=10)$ funds from the universe, and equally weight them to create a portfolio. Create 1,000 such randomly generated portfolios.

2. Select the year 2002 .

3. Calculate the annual return and record whether it is above or below the benchmark for that year for each of the 1,000 portfolios.

4. Determine the average, the 5th and 95th percentile of 1,000 portfolios excess returns.

5. Repeat steps 3 and 4 for each of the remaining years from 2003 to 2006.

6. Repeat steps 1-5 for different values of $M$.

\section{Strategy diversification}

As pointed out in the section 'Universe' the universe is skewed by long-short managers (representing 42 per cent of the total funds in the universe), and if we construct equal-weighted portfolios by simple random sampling, most of the portfolio performance will be dominated by long/short managers. A typical absolute return focused fund-of-funds would diversify its investment across different strategies. In order to simulate diversified portfolios we use the technique of stratified sampling.

The construction algorithm is exactly the same as the one described for simple random sampling except we require an equal number of funds to be drawn from each of the ten strategies. We construct portfolios of size $M$ (in multiples of 10) and draw $M / 10$ funds from each strategy. Since many of the strategies have only five funds, we choose a maximum $M$ equal to 50 . By imposing a constraint of equal investment in each strategy, we assume a simple strategy allocation of equal weighting.

\section{Simulation results}

In this section we discuss the results of the simulations. Using the procedures described in the previous section, we construct portfolios of different values of $M$. Figures 1 and 2 show returns at 95 and 5 per cent confidence levels as a function of portfolio size for years 2002, 2004 and 2006 (charts for the three years out of five years are shown for illustration). Table 4 tabulates the excess returns over the benchmark at a 95 per cent confidence level for portfolios with varying $M$. Figure 3 shows the scatter plot excess returns for portfolios of size 40 for the year 2006 .

Ninety-five per cent ( 5 per cent) confidence level is the same as the 5th (95th) percentile point. A randomly generated portfolio of same size $M$ has a 95 per cent probability of exceeding the 95 per cent confidence level return and a 5 per cent probability of exceeding the 5 per cent confidence level return.

\section{Naïve diversification versus strategy diversification}

Figures 1 and 2 show the 5 and 95 per cent confidence levels of excess returns as a function of $M$ using naïve diversification and strategy diversification methods, respectively. For a given $M$, if the 95 per cent confidence level line is above the $x$-axis (value becomes greater than zero) then it means that a randomly generated portfolio of the same size can beat the benchmark with 95 per cent probability. So for example in Figure 2 for the year 2004, a randomly selected portfolio of size 30 has a 95 per cent chance of beating the benchmark. For both the methods, the 95 per cent confidence level line increases with increasing $M$. What it means is that as $M$ increases, it becomes easier and easier to beat the benchmark.

The 95 per cent confidence level returns are also tabulated in Table 4 as a function of $M$ for all the years. As can be seen from Table 4(a), using the method of naive diversification, a portfolio of size 20 is able to beat the benchmark (corresponding to positive values) with 95 per cent probability for all years except for 2002 (a portfolio size of more than 150 is required to beat the benchmark as seen from Figure 1). The reason for poor performance is that 2002 was a bad year for long-short equity managers (see Table $3(\mathrm{~b})$ ), and since they occupy the majority of universe (42 per cent) this affects the performance of portfolios created by naive diversification. On the other hand, portfolios with size 40 generated by the method of strategy 

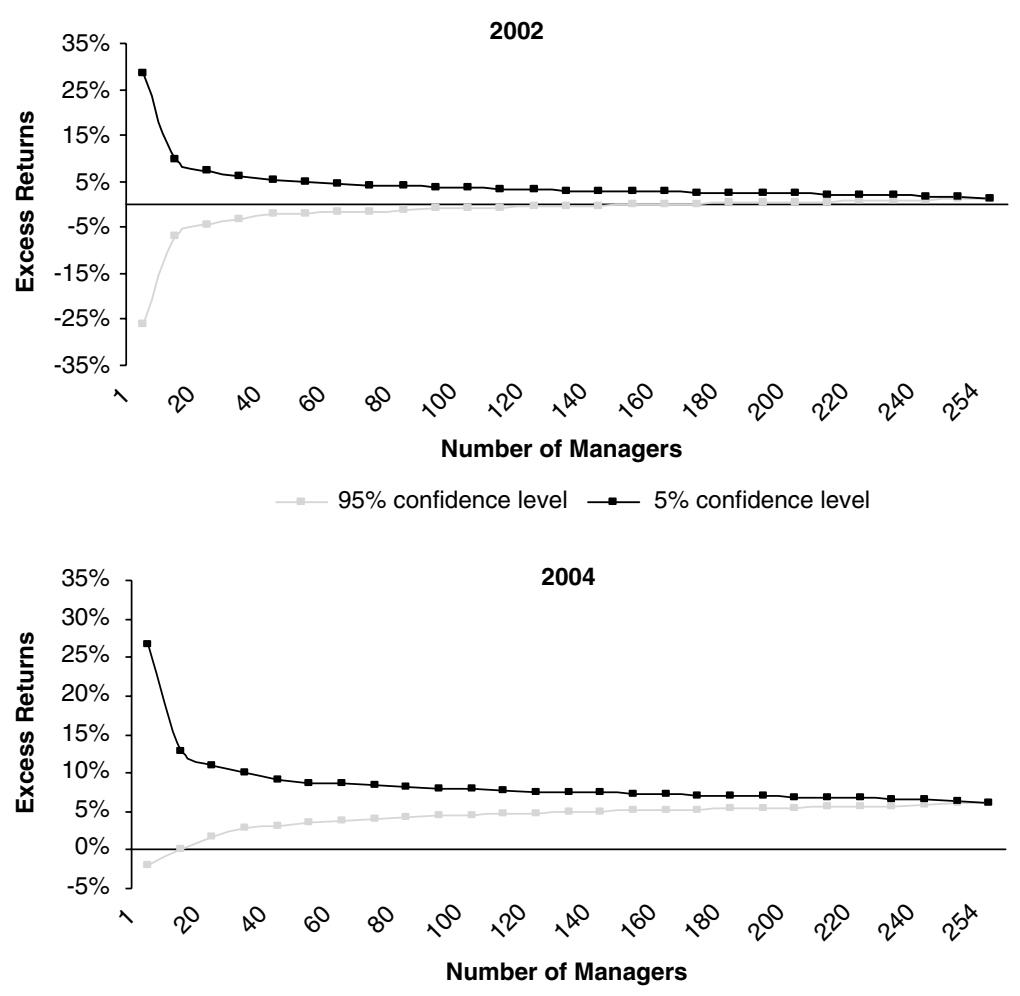

$95 \%$ confidence level $\rightarrow 5 \%$ confidence level

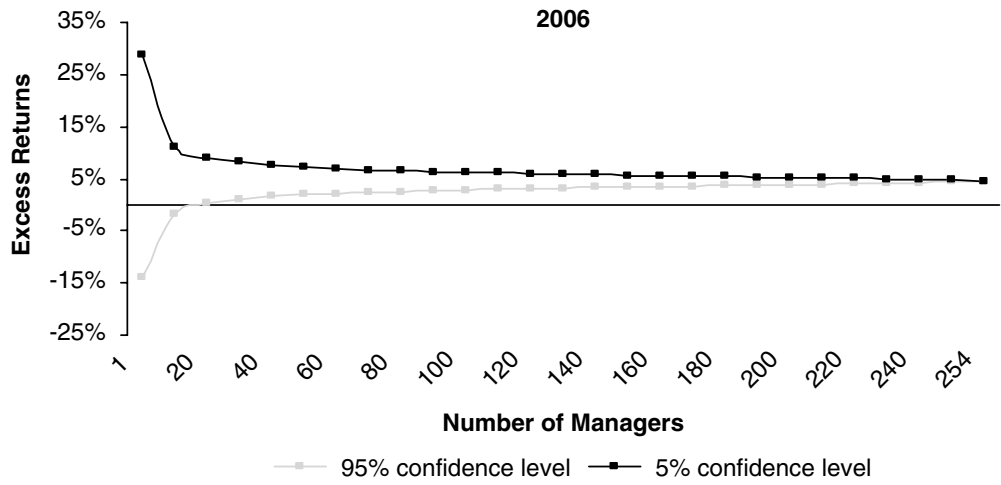

Figure 1: 95 and 5 per cent confidence levels as a function of size using naïve diversification

diversification are able to beat the benchmark (indicated by positive values in Table 4) in each of the five years with 95 per cent probability. The portfolio created by the method of strategy diversification equally weights different strategies, and is not affected by extreme under performance or over performance by any one strategy.

Figure 3 shows the scatter plot and histogram of portfolios of size 40 for the year 2006. As seen from the scatter plot, the average volatility of portfolios using strategy diversification method is much lower (3.1 per cent) than the average volatility using naïve diversification random sampling (5.4 per cent). The reason is strategy diversification offers more diversification (by equally weighting all the strategies) than naive diversification and thus results in a lower portfolio volatility on average.

\section{The limitation of diversification}

Figure 4 shows the probability density function of portfolios of size $M=20$ and 40 for 2006 using 

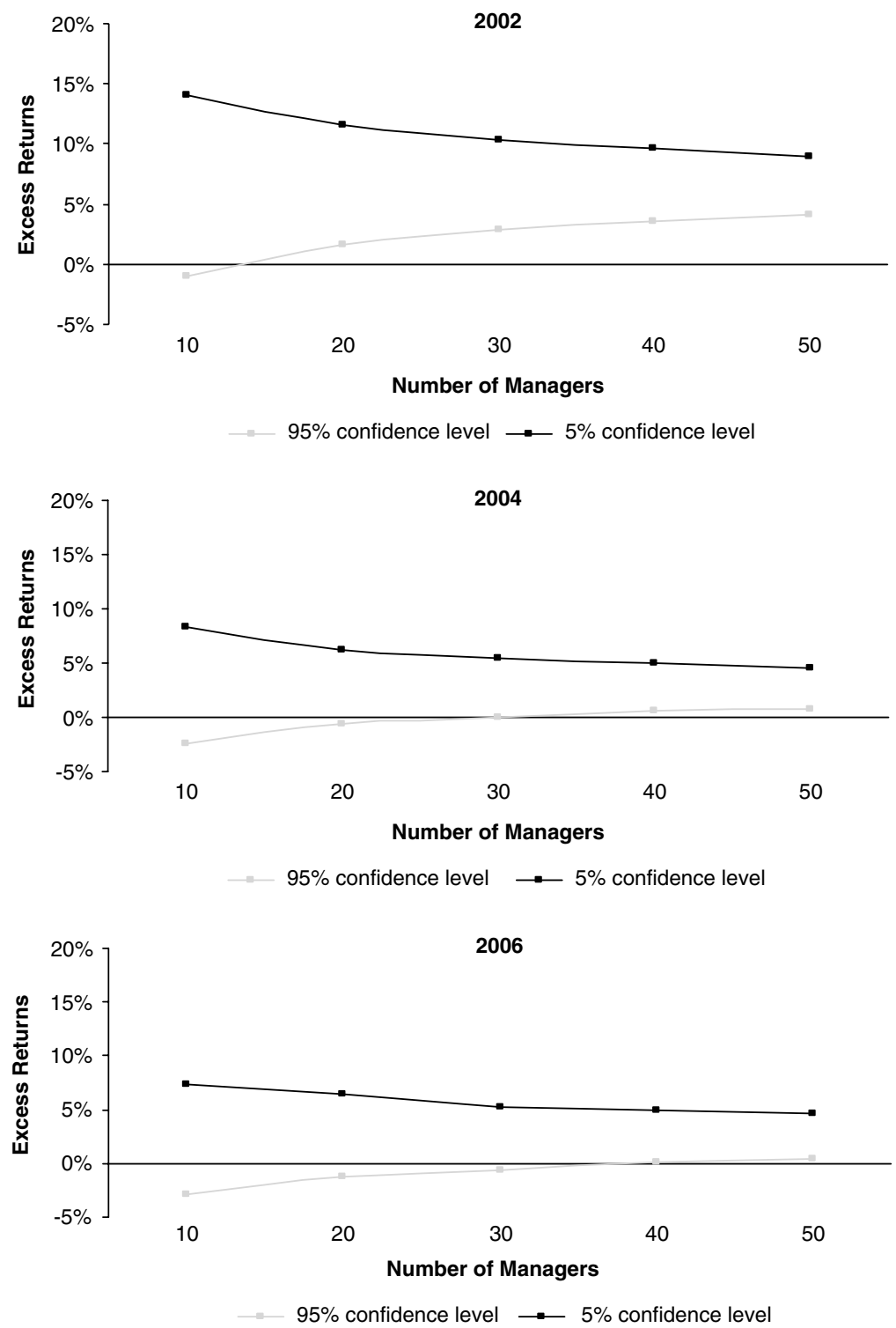

Figure 2: 95 and 5 per cent confidence levels as a function of size using strategy diversification

strategy diversification method. As can be seen from the figure, portfolios of size $M=20$ have a higher range of returns as compared to $M=40$ sized portfolios. Portfolios of size 40 have higher probability of outperforming the benchmark (measured by the area under the curve to the right of the benchmark line) as compared to portfolios of size $M=20$, but at the same time they have lower probability of exceeding benchmark +5 per cent returns (as measured by the area under the curve to the right of the benchmark +5 per cent line). A similar result can be seen from Figure 2 where the range of returns decreases with increasing $M$, which means that increasing the value of $M$ limits the maximum returns that a portfolio can achieve. Clearly the downside protection offered against the benchmark by a high value of $M$ comes with the cost of giving up higher returns that a lower $M$ can offer.

\section{Zero turnover portfolios - An extreme case}

So far we have focused on how different simulated portfolios perform relative to the benchmark on a year-to-year basis. Our analysis 
Table 4: Excess returns at 95\% confidence level for portfolios with different sizes

\begin{tabular}{lccccc}
\hline$M$ & $2002(\%)$ & $2003(\%)$ & $2004(\%)$ & 2005 (\%) & 2006 (\%) \\
\hline (a) Naïve diversification & & & & & \\
10 & -7.1 & 7.1 & 0.0 & 0.1 & -1.9 \\
20 & -4.3 & 9.9 & 1.7 & 3.3 & 0.2 \\
30 & -3.4 & 11.9 & 2.8 & 3.6 & 1.0 \\
40 & -2.0 & 12.5 & 3.1 & 3.9 & 1.9 \\
50 & -2.0 & 12.9 & 3.5 & 3.8 & 2.1 \\
60 & -1.8 & 13.6 & 3.8 & & \\
(b) Strategy diversification & & & & -1.6 & -2.9 \\
10 & -0.9 & 4.3 & -2.4 & -0.1 & -1.3 \\
20 & 1.6 & 6.6 & -0.7 & 0.7 & -0.6 \\
30 & 2.8 & 7.7 & 0.0 & 1.4 & 0.1 \\
40 & 3.6 & 8.6 & 0.6 & 0.8 & 0.4 \\
50 & 4.1 & 9.1 & & & \\
\hline
\end{tabular}
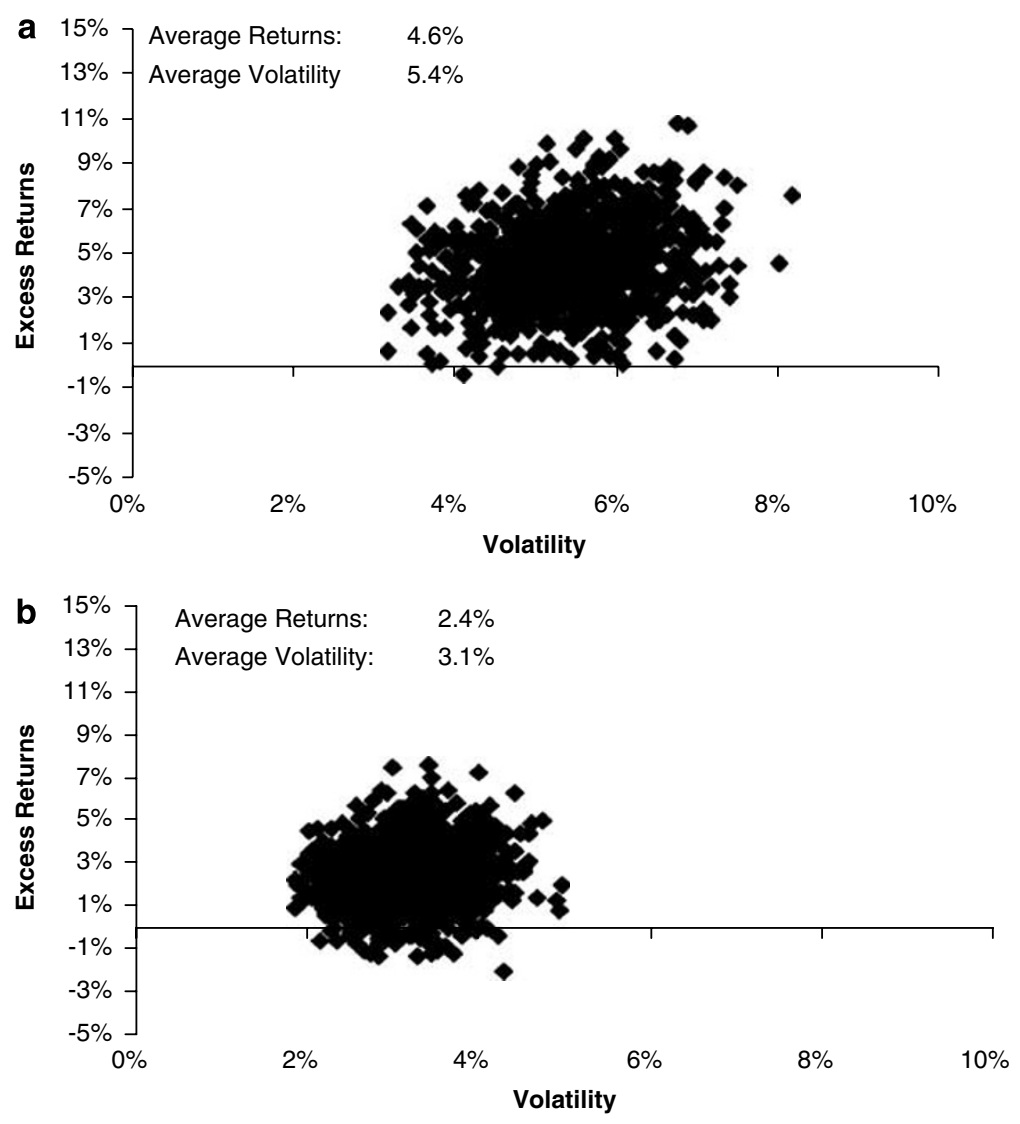

Figure 3: Scatter plots of portfolios of size $M=40$ for the year 2006: (a) naïve diversification and (b) strategy diversification

does not take into account the case when a portfolio beats the benchmark in one year but underperforms the benchmark in another year. In order to address the path dependence of performance of portfolios, we consider zero turnover portfolios. We analyse the risk and return performance of a zero turnover portfolio by taking a portfolio at the beginning of 2002 


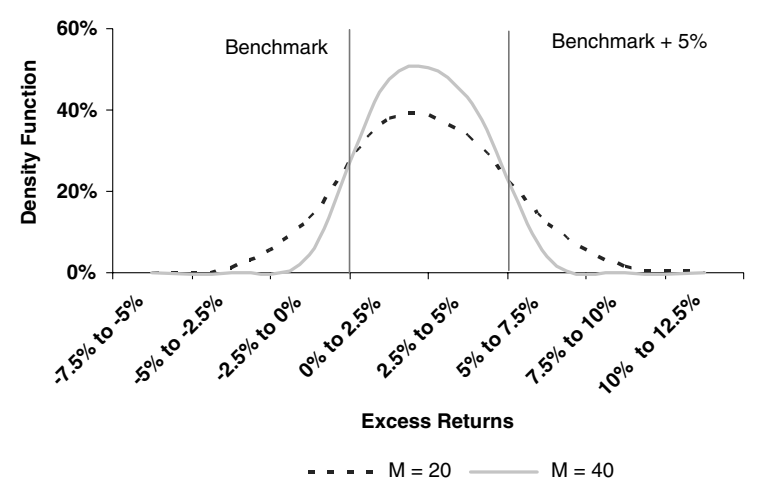

Figure 4: Portfolio returns density function for 2006

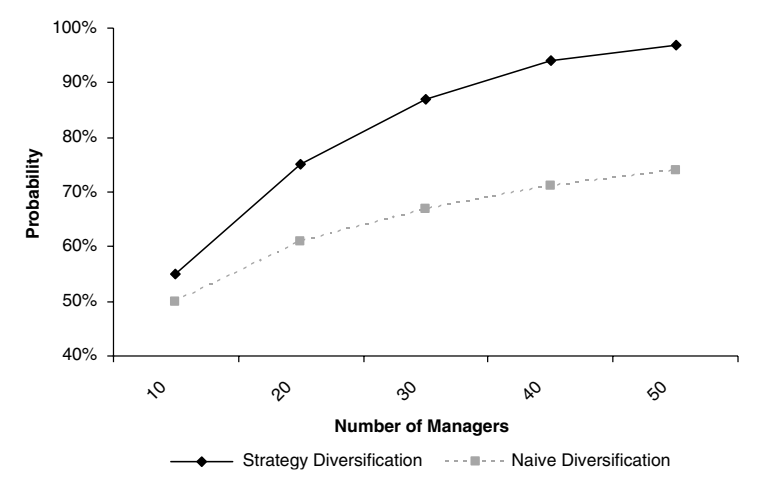

Figure 5: Probability of a zero turnover portfolio beating the benchmark in each of the consecutive years as a function of $M$

and holding its allocations constant for the entire period and comparing the returns with the benchmark for each year. ${ }^{9}$ The probability of portfolios beating the respective benchmarks in all the years is given in Figure 5 as a function of portfolio size. As can be seen from the figure, the probability of beating the benchmark using strategy diversification method exceeds that of portfolios generated using naïve diversification for all values of $M$. Also, portfolios of sizes 40 and 50 using strategy diversification method have a very high probability (94 and 97 per cent, respectively) of beating the benchmark.

In reality, a fund-of-funds won't have zero turnover for a period of five years. A fund-offunds management firm can enhance the performance of these constantly held portfolios, if it can correctly predict and move capital from underperforming managers (strategies) to outperforming managers (strategies).
Based on the results of individual year-by-year performance of portfolios and performance of zero turnover portfolios across all the years, we find that given our objective of beating the benchmark with a high confidence, 40 managers is an appropriate number to have in a portfolio, assuming a diversified fund-of-funds with an absolute return mandate.

\section{Conclusion}

Although portfolio construction is not typically based on the random selection of funds, our study of randomly generated portfolios illustrates the range of possible returns as a function of the number of managers. We believe portfolios generated by strategy diversification are representative of a typical diversified fund-offunds portfolio. For the universe, benchmark and the time period selected in our study, we conclude that a diversified portfolio of approximately size 40 is optimal for a fund-offunds portfolio. We, however, note that the following factors can influence the number of managers required.

1. Investment objective: In this paper we use a hypothetical benchmark (T-Bill +2.5 per cent) catering to the needs of an institutional investor. All else equal, increasing (decreasing) the required benchmark or the confidence level at which the return exceeds the benchmark will require a higher (lower) number of managers to be included in the portfolio.

2. Strategy allocation: We used a very simple strategy allocation (with all strategies equally weighted) to simulate a typical fund-of-funds portfolio. A strategy allocation based on correctly forecasting underperforming/outperforming strategies and allocating accordingly will achieve better performance than by equally weighting all strategies. All else equal, a better performing strategy allocation would necessitate a lower number of managers to be included in the portfolio.

3. Manager selection: Manager selection has a major impact on portfolio performance as illustrated in the study by Reddy et al. ${ }^{10}$ If a fund-offunds manager is able to predict and overweight 
outperforming managers, better performance can be achieved. Superior manager selection would imply a portfolio with a lower number of managers could still meet the benchmark.

The topic of optimal number of hedge funds required in a portfolio has been studied over the past several years. The number of optimal funds as suggested from other studies range from as low as 5 to 20 funds. L'habitant and Learned ${ }^{4}$ concludes that no more than 5-10 funds are required to diversify the portfolio risk away, Amo et al. ${ }^{5}$ suggests that the marginal benefit of adding a new fund decreases beyond six funds. Amin and Kat ${ }^{11}$ concludes that more than 15 funds is not necessary to have in a portfolio.

The reason why our research shows a different number of optimal managers can be attributed to one or all of the following factors: the starting universe, risk criteria and simulation methodology. We use the manager set represented in CS/ Tremont hedge fund index as our starting universe (which we believe is representative of funds that institutional investors would invest in), while other research mainly use larger databases (in which a majority of the funds have AUM less than $\$ 50 \mathrm{~m}$, typically not considered investable by institutional investors). The main risk we consider to determine optimal number of managers is that of underperforming a benchmark typically mandated by institutional investors, while other research studies use statistical return parameters such as volatility, correlation, drawdown, etc. Almost all research use only naïve diversification to simulate portfolio returns. Along with naïve diversification, we also use strategy diversification that we believe is more representative of a diversified fund-of-funds portfolio.

\section{Acknowledgments}

The author would like to thank Girish Reddy, Emanuel Derman, Peter Brady and Francis Conroy for their contribution to this paper. The opinions expressed in the paper are solely those of the author.

\section{References and Notes}

1 http://hedge.preqin.com/.

2 Markowitz, H. M. (1952) 'Portfolio selection', The Journal of Finance, Vol. 7, pp. 77-91.

3 Statman, M. (1987) 'How many stocks make a diversified portfolio?', Journal of Financial and Quantitative Analysis, Vol. 22, pp. 353-363.

4 L'habitant, F. S. and Learned, M. (2002) 'Hedge fund diversification: How much is enough?', The Journal of Alternative Investments, Vol. 5, No. 3, pp. 23-49.

5 Amo, A., Harasty, H. and Hillion, P. (2007) 'Diversification benefits of fund of hedge funds: Identifying the optimal number of hedge funds', The Journal of Alternative Investments, Vol. 10, No. 2, pp. 10-22.

6 An example of a portable alpha trade by an institutional investor is an initial investment in a fund-of-funds manager (alpha source), and a simultaneous entry into a total return swap contract of a desired market index (beta source) whereby the investor agrees to periodically pay returns of T-Bill+spread in exchange for index returns. This means that the responsibility of achieving T-Bill returns falls on the fund-of-funds manager.

7 The methodology of index rules is available on the following website: http://www.hedgeindex.com.

8 There is a survivorship bias introduced here because we consider only the funds that have entire five-year history. However, as part of our study we look at the return and risk characteristics of portfolios with zero turnover from year 2002 to 2006 , in which case this requirement (of five-year track record) of inclusion in the universe becomes important.

9 The probability of successful consecutive outcomes of an event would be less than probability of individual successes. For example, if we consider two tosses of a fair coin, the probability of getting a heads on the first toss (or the second toss) is 0.5 . However, the probability of getting two heads consecutively is $0.25(0.5 \times 0.5)$.

10 Reddy, G., Brady, P. and Patel, K. (2007) 'Are fund of funds simply multi-strategy managers with extra fees?', The Journal of Alternative Investments, Vol. 10, No. 3, pp. 49-61.

11 Amin, G. and Kat, H. M. (2002) 'Portfolios of hedge funds: What investors really invest in?', Working paper, ISMA University of Reading. 